\title{
Kinetics of Sodium and Selenium Reactions in Sodium Ion Batteries
}

\author{
Qianqian $\mathrm{Li}^{1}$, Heguang Liu ${ }^{2}$, Zhenpeng $\mathrm{Yao}^{1}$, Chris Wolverton ${ }^{1}$, Jinsong Wu*, Vinayak P Dravid ${ }^{1 *}$ \\ 1. Department of Materials Science and Engineering, NUANCE Center, Northwestern University, \\ Evanston, Illinois 60208, United States \\ 2. Department of Materials Science and Engineering, Northwestern Polytechnical University, Xi'an, \\ Shaanxi 710072, China
}

*Correspondence to: jinsong-wu@northwestern.edu; v-dravid@northwestern.edu

Selenium and sulfur, both as chalcogen elements, show similar volumetric capacity as cathode material for both lithium and sodium ion batteries.[1] Additionally selenium has notable higher electrical conductivity than sulfur.[2] In this work, we have investigated the kinetics of sodiation reaction in selenium nanotube as the cathode material for sodium ion battery. We have monitored the microstructure evolution and interface dynamics using in situ TEM during sodiation process. A three steps reaction mechanism appears to explain the sodiation process (Figure 1). In the first step, single crystalline selenium nanotube rapidly transforms to an amorphous $\mathrm{Na}_{\mathrm{x}} \mathrm{Se}$ alloy phase. In the second step with continued charging, the amorphous phase recrystallizes to a polycrystal $\mathrm{Na}_{2} \mathrm{Se}_{2}$ phase. In the final step near full sodiation, polycrystalline $\mathrm{Na}_{2} \mathrm{Se}_{2}$ appears to completely transform into $\mathrm{Na}_{2} \mathrm{Se}$ phase with high content of $\mathrm{Na}$. Intriguingly, the reaction front region movement is found to be quite different in the different sodiation stages. The solid-state amorphization process quickly finishes due to the high diffusion of sodium ions inside Se nanotube, with the highest nominal speed of $\sim 2.8 \mathrm{~nm} / \mathrm{s}$, and the recrystallization processes has a speed of $\sim 1.0 \mathrm{~nm} / \mathrm{s}$ (Figure 2). Moreover the speed of solid-state amorphization process is nearly 10 times higher than lithation process when selenium nanotube were tested in lithiation reaction. Molecule Dynamics (MD) calculation shows all the intermediate phases produced in sodiation are good conductor of both electrons and ions. These observations can not only reveal the reaction mechanism and reaction process, but also to provide insights to design novel nanostructure of electrodes with excellent electrochemical performance.

\section{References:}

[1] Abouimrane, A.; Dambournet, D.; Chapman, K. W. et al, Journal of American Chemical Society 132 (2012), 4505-4508

[2] Cui, Y.; Abouimrane, A.; Lu, J.; Bolin, T. et al, Journal of the American Chemical Society 135 (2013), 8047-8056.

[3] Acknowledgements: This work was supported as part of the Center for Electrochemical Energy Science, an Energy Frontier Research Center funded by the U.S. Department of Energy, Office of Science, Basic Energy Sciences under Award \# DEAC02-06CH11357, and the Initiative for Sustainability and Energy at Northwestern (ISEN). This work was also supported by the NUANCE Center, and made use of the EPIC facility (NUANCE Center-Northwestern University), which has received support from the MRSEC program (NSF DMR-1121262) at the Materials Research Center; the International Institute for Nanotechnology (IIN); and the State of Illinois, through the IIN. 


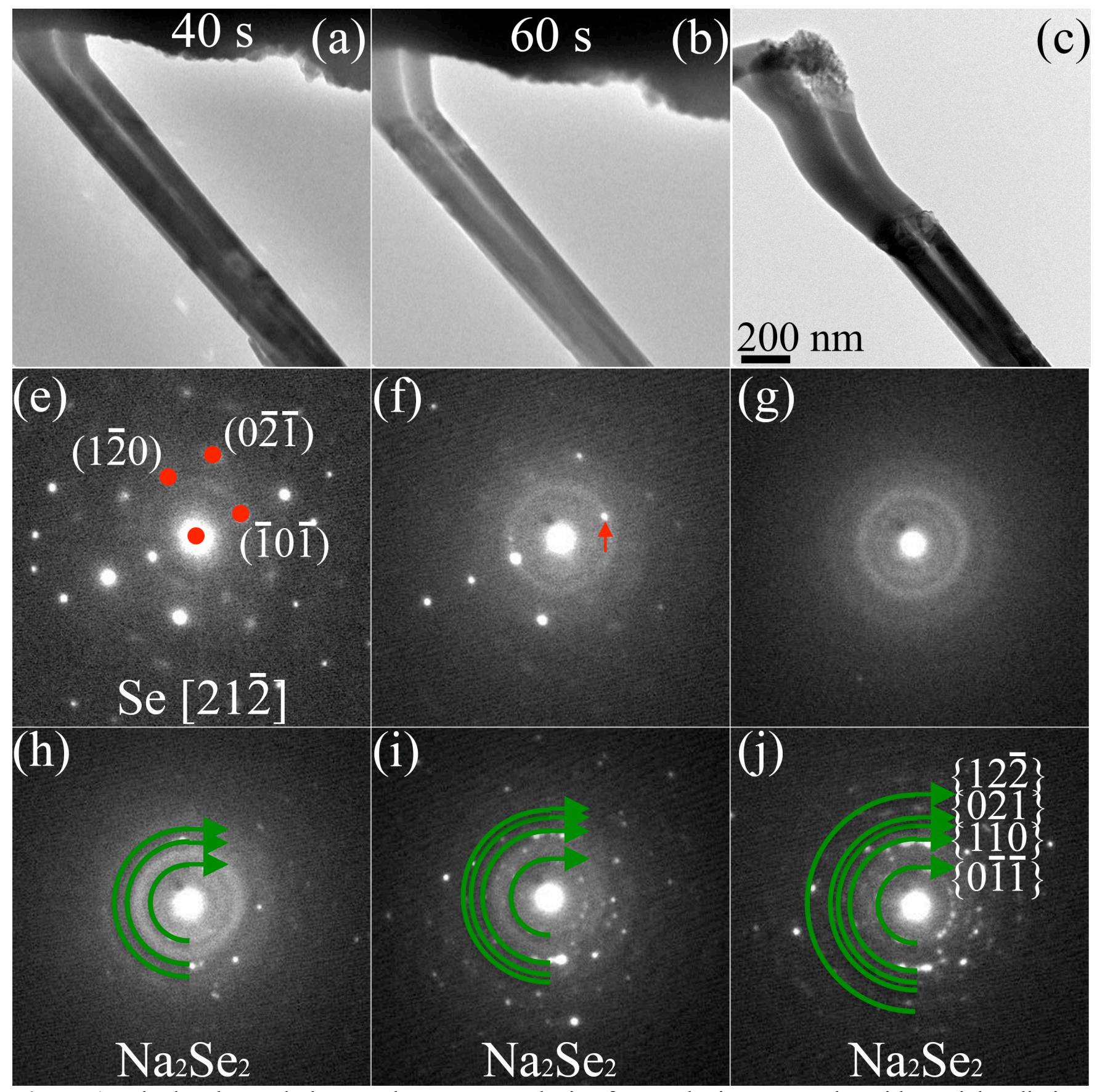

Figure 1. The local morphology and structure analysis of one selenium nanotube with partial sodiation. (a-b) In-situ sodiation process of the one single selenium nanotube. (c) The morphology of the partially sodiated selenium nanotube. (e-j) The electron diffraction patterns are taken from the different phase region and the interface between two adjacent phases. The partially sodiated selenium nanotube shows the three sodiation stages in one nanotube. The unreacted region, amorphous region and the recrystallized region. 


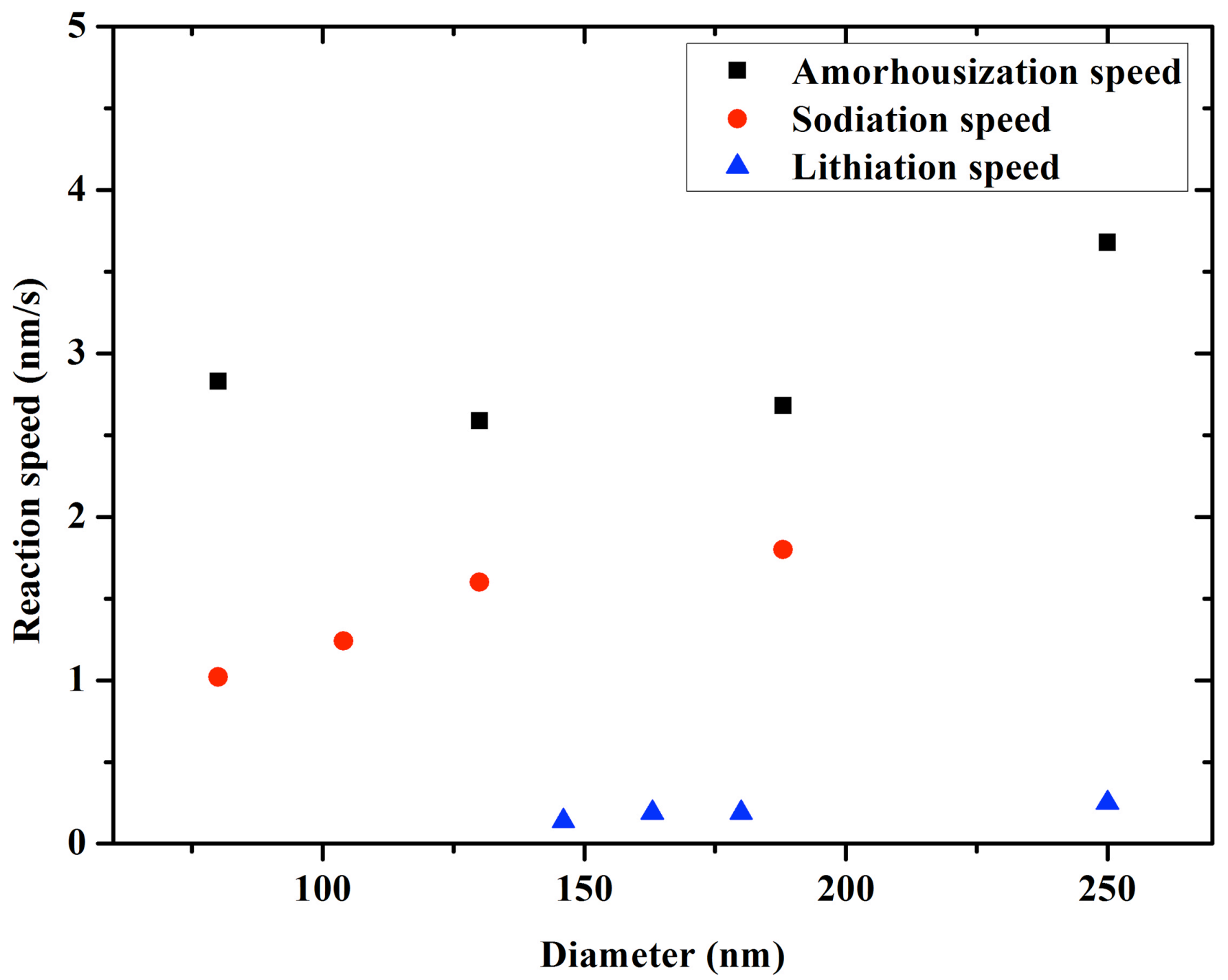

Figure 2. The reaction speed of different diameter selenium nanotubes. The solid-state amorphization stage with the highest reaction speed of $\sim 2.8 \mathrm{~nm} / \mathrm{s}$; and the total speed of sodiaton process has a speed of $\sim 1.3 \mathrm{~nm} / \mathrm{s}$, due to the lower speed of recrystallization stage in the whole sodiation process. The average lithiation speed is $\sim 0.23 \mathrm{~nm} / \mathrm{s}$. 
https://doi.org/10.1017/S1431927616004980 Published online by Cambridge University Press 